\title{
Current sheet flapping motion and structure observed by Cluster
}

\author{
V. Sergeev, ${ }^{1}$ A. Runov, ${ }^{2}$ W. Baumjohann, ${ }^{2}$ R. Nakamura, ${ }^{2}$ T. L. Zhang, ${ }^{2}$ M. Volwerk, ${ }^{2}$ \\ A. Balogh, ${ }^{3}$ H. Rème, ${ }^{4}$ J. A. Sauvaud, ${ }^{4}$ M. André, ${ }^{5}$ and B. Klecker ${ }^{6}$ \\ Received 23 October 2002; revised 18 December 2002; accepted 2 January 2003; published 27 March 2003.
}

[1] Fast vertical flapping oscillations of the plasma sheet have been observed by Cluster on September 26, 2001. The flapping motion had vertical speeds exceeding $100 \mathrm{~km} / \mathrm{s}$, an amplitude in excess of $1 R_{E}$ and a quasiperiod of $\sim 3 \mathrm{~min}$. The current sheet was mostly tilted in the $Y$ - $Z$ plane (with the tilt sometimes exceeding $45^{\circ}$ ). The waves had the properties of a kink mode and propagated toward the dusk flank. The flapping allowed to probe the vertical structure of the plasma sheet. Three different methods gave consistent evidence of a bifurcated structure of the cross-tail current with about half of all current concentrated in two sheets (each $\sim 500-1000$ $\mathrm{km}$ thick). The current density peaks at $\left|B_{x}\right| \sim 0.5 B_{L}$, with a pronounced current density minimum and a plasma density plateau between these peaks. INDEX TERMS: 2744 Magnetospheric Physics: Magnetotail; 2764 Magnetospheric Physics: Plasma sheet; 2760 Magnetospheric Physics: Plasma convection. Citation: Sergeev, V., et al., Current sheet flapping motion and structure observed by Cluster, Geophys. Res. Lett., 30(6), 1327, doi:10.1029/2002GL016500, 2003.

\section{Introduction}

[2] Stability properties of space current sheets (CS) depend on the sheet parameters as well as on how the equilibrium is established. The most well-known 1-d current sheet model has a broad maximum of current density in the sheet center. Other possibilities can include a strong concentration of the current in the central region due to the ion inertia forces in an anisotropic plasma [Sitnov et al., 2000], or more complicated distributions with double off-center peaks. Such bifurcated current sheet, for example, can be born in the plasma outflow region when the magnetic reconnection process is operating [e.g., Arzner and Scholer, 2001]. Whether and where bifurcated current sheets really occur in space, and whether they are an important constituent of the explosive dissipative process responsible for the substorms, are still open questions.

[3] The observational evidence for bifurcated current sheets is yet very scarce. Previous studies with ISEE-1/2 dual s/c system gave only one example of a bifurcated thin current sheet [Sergeev et al., 1993]. Other arguments for the existence of bifurcated CS have been given by Hoshino et al. [1996] although they refer to the distant tail plasma sheet, where the properties are different from those in the

\footnotetext{
${ }^{1}$ Institute of Physics, St. Petersburg University, St. Petersburg, Russia.

${ }^{2}$ Institut für Weltraumforschung der ÖAW, Graz, Austria.

${ }^{3}$ Imperial College, London, UK.

${ }^{4}$ CESR/CNRS, Toulouse, France.

${ }^{5}$ Swedish Institute of Space Physics, Uppsala, Sweden.

${ }^{6}$ Max-Planck-Institut für extraterrestrische Physik, Garching, Germany.
}

near- and mid-tail regions. Further evidence of current bifurcation has been recently given by Asano [2001] who statistically analysed current densities obtained from ion and electron velocities measured by the Geotail spacecraft.

[4] Reconstruction of the spatial profile of plasma parameters (including electric current) in the time-varying plasma sheet can be done with Cluster observations if the spacecraft repeatedly crosses the current sheet. This occurs when the vertical oscillating motions of the plasma sheet (flapping motions) have a large amplitude. The origin and the nature of such waves also have not yet been established, although they carry important information on the processes destabilizing the current sheet. Because of difficulties to study them with a single s/c there are only few attempts [e.g., Toyichi and Miyasaki, 1976; Bauer et al., 1995; Sergeev et al., 1998] yet with no consistent mode identification.

\section{Observations and Data Analysis}

[5] At 22 UT on September 26, 2001, Cluster was located at $[-18 ;+7 ; 0] R_{E}$ (in GSM coordinates, used everywhere if not mentioned otherwise) near the plasma sheet northern boundary. The IMF was mostly northward during preceeding hours, so the magnetic activity was low. Distinct sudden activation of the westward auroral electrojet was recorded at 2223 UT near midnight in the center of the IMAGE groundbased network, with maximum negative magnetic bay amplitude $(\sim 140 \mathrm{nT})$ at the MAS station (Figure 1). The quicklook of the WIC camera on the IMAGE satellite confirmed that the auroral brightening occurred at 2223 UT in the 21-24 MLT sector. Pi2 pulsation onset was recorded at 2224 UT on subauroral stations of the SAMNET network (not shown). Another feature of interest in the context of our study is a factor of 2 drop of solar wind density (and dynamic pressure, $\mathrm{P}_{\mathrm{D}}$ ). It was later recorded as a sudden negative impulse (-SI in Figure 1) at 2216 UT by GOES-10 in the dayside magnetosphere. The onset of intense flapping motion at $X \sim-18 R_{E}$ was observed by the Cluster s/c after 2218 UT, a few minutes before the ground onset of substorm activation. Five $\mathrm{s} / \mathrm{c}$ entries to the plasma sheet from the lobe were observed while the first (at $\sim 2218$ UT) not of interest as three of the four $\mathrm{s} / \mathrm{c}$ stayed in the lobe.

[6] Figure 2 displays the detailed view of observations during the oscillations $\mathrm{A}$ to $\mathrm{C}$ which have an apparent period of $\sim 3 \mathrm{~min}$. The upper plot shows an intercomparison between the $E_{y}$ electric field component measured by the EFW instrument (double probe) [Gustafsson et al., 2001] and the $y$-component of $\mathbf{V}_{p} \times \mathbf{B}\left(\mathbf{V}_{\mathbf{p}}\right.$ is the proton velocity from CIS-1 mass spectrometer [Réme et al., 2001], $\mathbf{B}$ is the magnetic field vector from [Balogh et al., 2001]).

[7] Being confident in the quality of plasma measurements, we now integrate $V_{p_{z}}$ to visualize the flapping oscillation and to get an estimate of its vertical scale. 


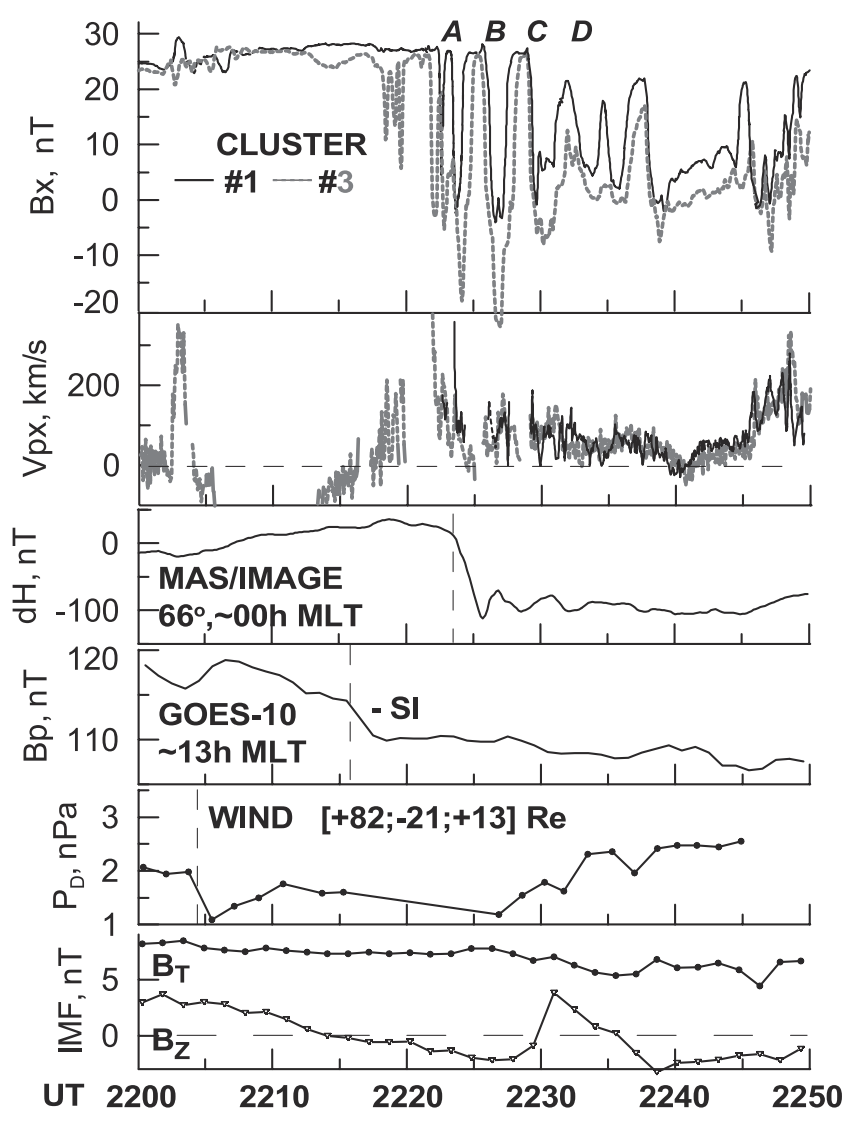

Figure 1. Sept. 26, 2001: Overview of observations in the plasma sheet, auroral ionosphere, dayside magnetosphere and solar wind. WIND data have been time shifted at solar wind velocity to $X=10 R_{E}$.

Indeed, this gives a similar variation at both $\mathrm{s} / \mathrm{c} \# 1$ and \#3 (Figure $2 \mathrm{~b}$ ) consistent with the flapping-related $B_{x}$ variation (Figure 2c). It also shows a clear trend: it is larger at $\mathrm{s} / \mathrm{c} \# 1$, which stayed above the neutral sheet and can be interpreted as contribution from the convection plus some spurious effects. In the following analysis we use detrended data with the trend computed over the time interval from 2216 UT till $2250 \mathrm{UT}$, which had slopes of $21.5 \mathrm{~km} / \mathrm{s}$ and $16.8 \mathrm{~km} / \mathrm{s}$ at s/ $\mathrm{c} \# 1$ and \#3. This will be referred to as the vertical distance $Z^{*}$ and used below in the Figure 3 .

[8] To analyse the flapping oscillation in more detail we use inter-s/c timing as well as Minimum Variance Analysis. The MVA in our case was applied to the short (15-30 s) time intervals during which the $\mathrm{s} / \mathrm{c}$ crossed from the lobe boundary to the neutral sheet or vice versa. They characterize a part of the upward/downward phase of the current sheet oscillatory motion in either the northern half (data from all s/c) or the southern half (only \#3 provides data) of the current sheet. 20 of 21 MVA determinations gave wellresolved MVA normals (with intermediate-to-minimal eigenvalue ratio exceeding 3 ). Their projections to the $Y-Z$ plane are displayed in Figure 2d, the X-component of the normal is always smaller as compared to $n_{Y}$ and $n_{Z}$, so the tilt in $X-Z$ plane is insignificant.

[9] The most consistent results come from data of s/c \#3, which crossed the entire sheet between the two lobes. It shows the opposite $Y$-slopes at upward/downward phases of the flapping oscillation, which have the same sense in the northern and southern halves of the current sheet for each phase. The same sense of sheet tilt in northern and southern regions implies that the sheet is tilted as a whole (suggesting kink mode) rather than being locally expanded and contracted (like in the sausage wave). Other s/c show typically smaller slopes of the normal (probing only part of the oscillations), in many cases their average (except for upward phases of oscillations A and B) are of the same sense and agree with s/c \#3.

[10] The timing of the $B_{x}$ component records at four s/c (Figure 2c) confirms essentially this MVA-based picture. First, it shows a well-ordered vertical structure of the current sheet $\left(B_{X_{3}}\right.$ is always much smaller than $B_{X_{1}}$ if not in the lobes, consistent with s/c \#3 being at smaller $Z$ than other three s/c, see Figure 1). For other s/c having nearly the same $Z$ coordinate, the variations are similar with a small time delay between \#1 and \#4 but a large delay between those and $\mathrm{s} / \mathrm{c} \# 2$. This agrees with the sheet tilting mostly in the $Y$ $Z$ plane. The time delay from \#1, 4 to $\# 2$ varied between $23 \mathrm{~s}$ and $41 \mathrm{~s}$ for the upward and downward parts of oscillations $\mathrm{A}, \mathrm{B}$, and $\mathrm{C}$, which corresponds to a duskward propagation of the large-scale wave with a wavelength in $Y$-direction about $2 R_{E}$. This length is comparable to the vertical flapping amplitude which is $\sim 1 R_{E}$ from Figure $2 \mathrm{~b}$ (if subtracting the trend). The largest tilt of the sheet in $Y-Z$ plane was observed for oscillation $\mathrm{B}\left(60^{\circ}-75^{\circ}\right.$ according to s/c \#3 data). This is consistent with other sources: it has the

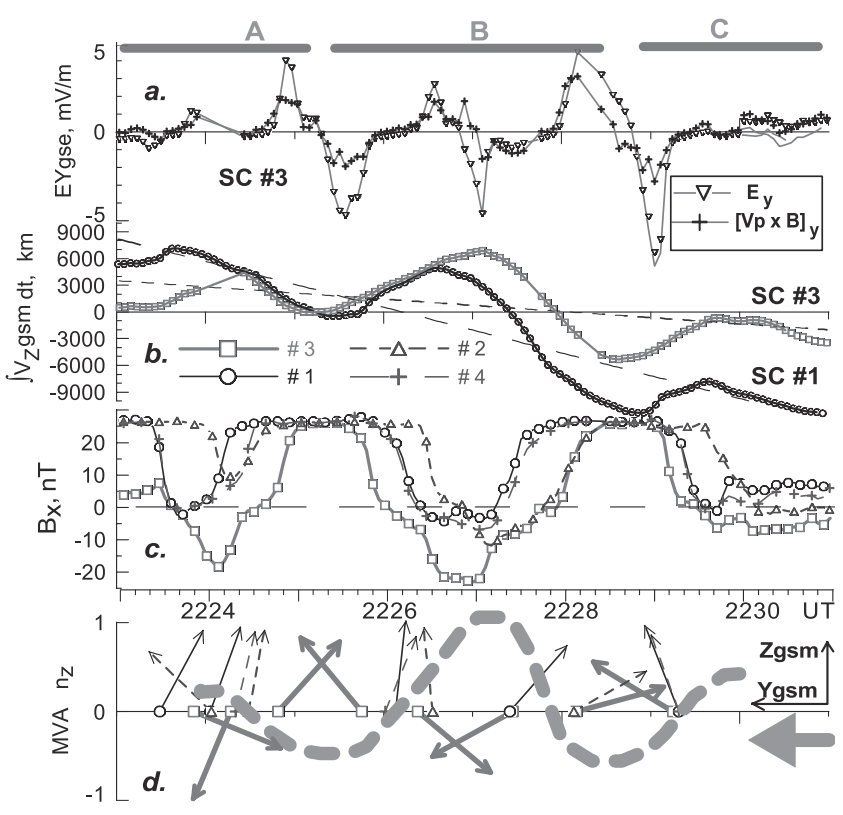

Figure 2. Observations during complete flapping oscillations A-C. (a) Comparison of $y_{G S E}$-components of $\mathrm{E}$ (from EFW instrument) and $\left[\mathbf{V}_{p} \times \mathbf{B}\right]$ (from CODIF and MGF) at the s/c \#3; (b) time integral of GSM ion bulk flow $\mathrm{z}$ component $V_{p_{z}}$; (c) variations of $B_{x}$-components at four s/c (every 3rd data point is marked); (d) $Y-Z$ projections of the MVA the current sheet normals determined during upward and downward phases of flapping. The thick dashed line is a rough sketch of the wavy neutral sheet geometry based on the computed normal directions. 

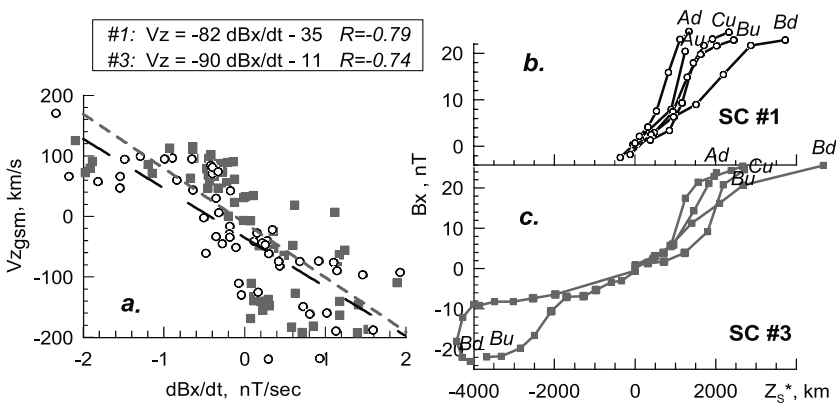

Figure 3. (a) Relationship between $V_{z}$ and $d B_{x} / d t$ in the flapping current sheet, $(\mathrm{b}, \mathrm{c}) B_{x}$ profiles vs. the $Z^{*}$ scale obtained by $V_{z}$ integration for individual crossings, across the neutral sheet (where $Z^{*}=0$ ). Letters A, B, C denote the individual oscillations whereas $\mathrm{u}, \mathrm{d}$ correspond to downward and upward halves.

largest flapping amplitude in $Z^{*}$ (Figure 2b) and largest difference in time delay between $\# 1$ and $\# 2$.

[11] Figure 3 provides some information to evaluate the current sheet thickness. According to Sergeev et al., [1998] in the case of good linear correlation between $V_{z}$ and $d B_{x} / d t$ the regression coefficient $v$ gives information about the average gradient $d B_{x} / d z$, allowing to infer the current density and current sheet scale. Figure 3 a demonstrate a good anticorrelation (correlation coefficients -0.79 and $-0.74)$ between these quantities. The average slope of $\sim 85 \mathrm{~km} / \mathrm{nT}$ corresponds to of $\sim 9.4 \mathrm{nA} / \mathrm{m}^{2}$, and the average CS half-thickness $\left(h a \equiv B_{L} /\left(d B_{x} / d z\right)=B_{L} \nu\right)$ appears to be $h a$ $\sim 2300 \mathrm{~km}=0.36 R_{E}$ (with $B_{L}=27 \mathrm{nT}$ ). Another method could use the apparent distance of $\mathrm{s} / \mathrm{c}$ from the neutral sheet $Z_{S}^{*}=Z_{0}-\int V_{z} d t$ (with subtracted long-term trend, as mentioned above, and $Z_{0}$ chosen to get $Z_{S}^{*}=0$ at the neutral sheet, where $B_{x}=0$ ). Data from both $\mathrm{s} / \mathrm{c}$ (\#1 and \#3 in Figures $3 \mathrm{~b}$ and $3 \mathrm{c}$ ) show remarkable similarity in all individual crossings: they indicate a reduced $d B_{x} / d z$ in the central part of the current sheet (where $B_{x} \ll B_{L}$ ), and enhanced gradient (enhanced current density) concentrated in the boundary part (in most cases between $B_{x}=10 \mathrm{nT}$ and $20 \mathrm{nT}$ ). The half-thickness of the whole sheet is comparable to the average above obtained $(2300 \mathrm{~km})$, with the thickness of concentrated current $\sim 500$ to $1000 \mathrm{~km}$ and maximal current densities of $20 \mathrm{nA} / \mathrm{m}^{2}$. These values give the upper limit of the actual CS thickness as the method ignores the tilt $\Psi$ of the current sheet in $Y-Z$ plane which could be quite significant.

[12] Following the approach by Sergeev et al. [1993] and assuming a $1 \mathrm{D}$ current sheet, we use the difference $d B_{x}=$ $B_{x_{1}}-B_{x_{3}}$ of the $X$-components measured by s/c \#1 and \#3, which gives us the total current in the horizontal slab between two s/c. With the s/c separation $d Z_{13}$ it gives the current density simply as $j_{y}=d B_{x_{13}} /\left(\mu_{0} d Z_{13} \cos \Psi\right)$, where $\Psi$ is the angle between $Z$-axis and the normal to the sheet. As a label of position in the current sheet where this current density has to be assigned, we use the $B_{x}$ component value in the mid point, that is $B_{x_{a}}=0.5\left(B_{x_{3}}+B_{x_{1}}\right)$. This gives us the relative vertical distance (with $B_{x}=0$ at the center).

[13] Hodograms of $d B_{x}$ versus $B_{x_{a}}$ at the top of Figure 4 show that initially (crossing A, showing most severe and complicated variations) the dynamics is very complicated, being possibly dominated by transient and/or wave features. Then, starting from the most deep plasma sheet crossing B (after 2225:20 UT) the trajectory displays a systematic behaviour, with a deep local minimum in the central part (where $B_{x} \sim 0$ ) and the maximum $d B_{x}$ at $\left|B_{x}\right| \sim 15 \mathrm{nT}$. This behaviour is repeated in the crossings $\mathrm{C}, \mathrm{D}$, so this bifurcated current structure was nearly stable for the next $\sim 15$ minutes. The $d B_{x}$ value at the current maximum evolved from $\sim 25 \mathrm{nT}$ (B) to $\sim 17 \mathrm{nT}$ (C) and $\sim 12 \mathrm{nT}(\mathrm{D})$, consistent with the $\sim 2$ times current sheet broadening during that time interval. Note that the $20 \mathrm{nT}$ difference at the large separation $d Z_{13}=1560 \mathrm{~km}$ gives $\sim 10 \mathrm{nA} / \mathrm{m}^{2}$, which is the lower limit for the current density in the maximum region. The bottom panel in Figure 4 shows the occurrence rate of magnetic field $B_{x}$ values; here we used the merged $1 \mathrm{~s}$ data from s/c \#1 and \#3 between 2220 and 2240 UT. As argued by Hoshino et al. [1996], in the case of constant flapping velocity $V_{z}$ the $B_{x}$-occurrence frequency is inversely proportional to the vertical $B_{x}$ gradients, so the peaks in the current density $\left(j_{y} \sim d B_{x} / d_{z}\right)$ should correspond to minima in the occurrence rate. Taking into account that the occurrence rate is skewed toward the northern lobe, where s/c \#1 stayed most of time (this trend is marked by the dashed line on the plot), this occurrence plot shows excellent agreement with the $d B_{z}$ vs. $B x_{a}$ profiles. Both methods confirm the findings

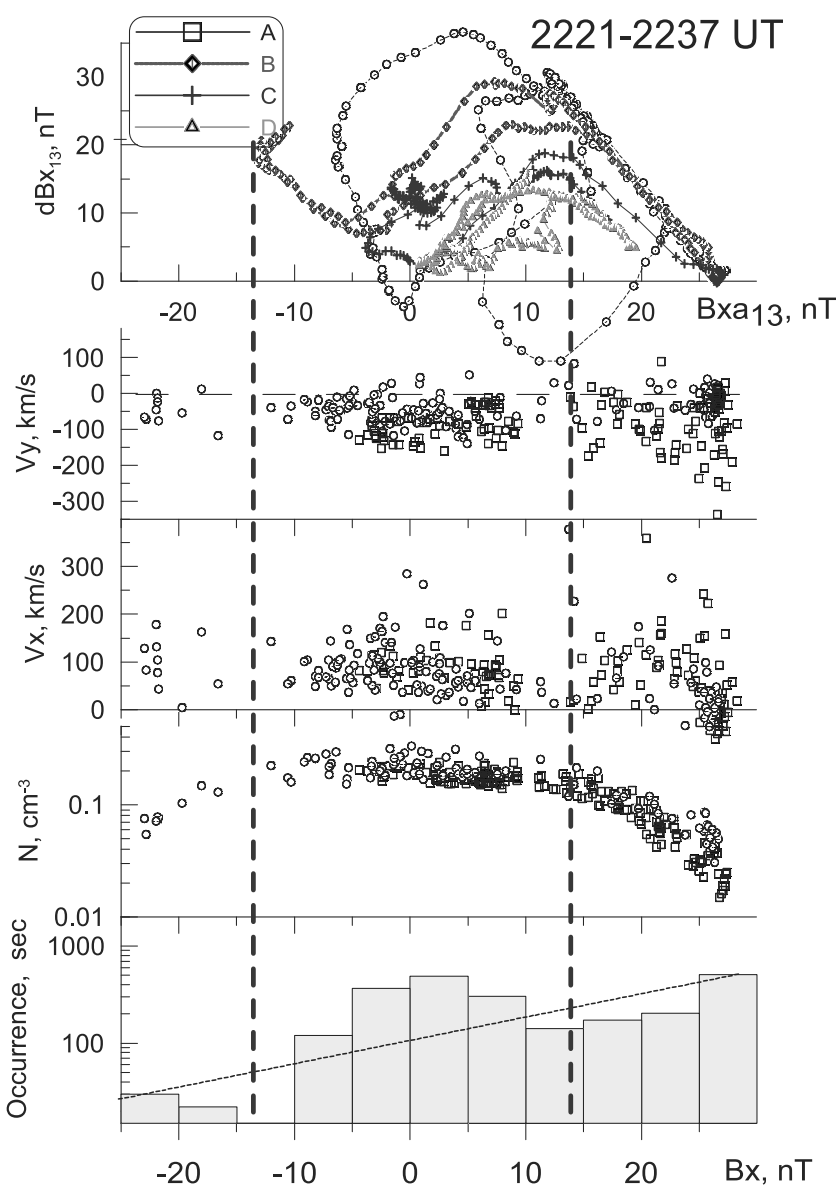

Figure 4. Distributions of plasma sheet parameters and magnetic field difference $\left(d B_{x_{13}}\right)$ vs. $B_{x}$ during 2221:002240:37 from s/c \#1 and \#3, and occurrence rate distribution of $B_{x}$. 
from Figure 3 and give a consistent picture of a stable bifurcated current, which peaks at $\left|B_{x}\right| \sim 0.5 B_{L}$ (lobe field was $\left.B_{L} \sim 27 \mathrm{nT}\right)$ and has a deep (2-3 times lower) broad valley in the central part of the current sheet.

[14] In the same way we constructed the vertical profiles of other parameters during the time period of bifurcated current, see also Figure 4. The most clear pattern was found for the density which shows a plateau in the broad central region between the peaks of bifurcated current as well as sharp decreases towards the lobe-plasma sheet boundary. No special features are noticed in $x$ - and $y$-components of the proton flow. Particularly, the dawnward $V_{y}$ (opposite to the direction of cross-tail current) shows no peculiar feature in the current maximum region. This may be an indication that the bifurcated current is supported by electrons. We failed to find the quadrupole $B_{y}$ perturbation near the current maximum region which is expected to be generated by the Hall currents near the reconnection region [e.g., Arzner and Scholer, 2001] and conclude that, if existed, it was too weak (less than a few nT) and masked by the strong noise.

\section{Discussion and Concluding Remarks}

[15] Main results of this study are the interesting properties of the flapping wave allowing to identify it as the kink mode, and reliable identification of the current bifurcation. Both results have considerable support in recent observations made also during substorms. Runov et al. [2002] reported MVA results indicating that the basic tilt of the plasma sheet in the flapping wave is in the $Y-Z$ plane, they also inferred that the basic propagation is in the Y-direction from the timing analysis at Cluster s/c. Our results extend these findings and also show the consistence of normals determined in northern and southern halves of the sheet with the kink mode character of the flapping wave.

[16] Signatures of bifurcated current were recently reported by Nakamura et al. [2002] in an onset-associated fast Earthward flow and by Runov et al. [2002] during a substorm activation. Double off-center peaks of the current density shown in our study (Figure 4, top) resemble the profiles inferred by Sergeev et al. [1993] (during substorm activation with strong Earthward flow pulse) and Runov et al. [2002], which also displayed a peak at $B_{x} \sim 0.5 B_{L}$ and a factor 2-3 difference between off-center current density peak and current minimum at the center. Our results extend these findings in that (1) three different methods gave the same results, and (2) the bifurcated current picture was found to be stable as it repeated a few times during $\sim 15 \mathrm{~min}$ of flapping wave observations.

[17] However unlike previous studies in our event there were no strong flows: $V_{p_{x}}$ rarely exceed $+200 \mathrm{~km} / \mathrm{s}$, which is much less than the expected Alfven speed in the lobes. Stagnant bifurcated current sheets have been sometimes met in the analysis made by Hoshino et al. [1996]. This feature makes it questionable that reconnection is the ultimate reason of the bifurcated structure. A process of a thin current sheet "aging", described by Zelenyi et al. [2002], may be an alternative mechanism of the CS bifurcation.

[18] Acknowledgments. The authors thank H.-U. Eichelberger for preparing the FGM data. WIND, GOES magnetometer data and quick-look IMAGE WIC data are available via CDAWEB. We thank the Finnish Meteorological Institute for the IMAGE and York University for the SAMNET magnetogram. V.A.S. thanks the IWF DEAW for support during his stay in Graz, as well as support by the RFBR grant N 03-02-17533 and from the Intergeophysics Program.

\section{References}

Arzner, K., and M. Scholer, Kinetic structure of the post plasmoid plasma sheet during magnetic reconnection, J. Geophys. Res., 106, 3827, 2001.

Asano, Y., Configuration of the thin current sheet in substorms, Ph.D. thesis, Univ. Tokyo, 2001.

Balogh, A., et al., The Cluster magnetic field investigation: overview of inflight performance and initial results, Ann. Geophys., 19, 1207, 2001.

Bauer, T. M., W. Baumjohann, and R. A. Treumann, Neutral sheet oscillations at substorm onset, J. Geophys. Res., 100, 23,737, 1995.

Gustafsson, G., et al., First results of electric field and density observations by Cluster/EFW based on initial months of operation, Ann. Geophys., 19, 1219-1240, 2001.

Hoshino, M., A. Nishida, T. Mukai, Y. Saito, and T. Yamamoto, Structure of plasma sheet in magnetotail: Double-peaked electric current sheet, J. Geophys. Res, 101, 24,775-24,786, 1996.

Nakamura, R., et al., Fast flow during current sheet thinning, in press, Geophys. Res. Lett., 2002.

Réme, H., et al., First multispacecraft ion measurements in and near the Earth's magnetosphere with the identical Cluster ion spectrometry (CIS) experiment, Ann. Geophys., 19, 1303, 2001.

Runov, A., R. Nakamura, W. Baumjohann, T. L. Zhang, M. Volwerk, H.-U. Eichelberger, and A. Balogh, Cluster observation of a bifurcated current sheet, Geophys. Res. Lett., submitted, 2002.

Sergeev, V. A., D. G. Mitchell, C. T. Russell, and D. J. Williams, Structure of the tail plasma/current sheet at $11 \mathrm{Re}$ and its changes in the course of a substorm, J. Geophys. Res., 98, 17,345, 1993.

Sergeev, V., V. Angelopulous, C. Carlson, and P. Sutcliffe, Current sheet measurements within a flapping plasma sheet, J. Geophys. Res., 103, 9177, 1998.

Sitnov, M. I., H. V. Malova, L. M. Zelenyi, and A. S. Sharma, Thin current sheet embedded within a thicker plasma sheet: Self-consistent kinetic theory, J. Geophys. Res., 105, 13,029, 2000.

Toyichi, T., and T. Miyazaki, Flapping motions of the tail plasma sheet induced by the interplanetary magnetic field variations, Planet. Space Sci., 24, 147, 1976.

Zelenyi, L., D. C. Delcourt, H. V. Malova, and A. S. Sharma, "Aging” of the magnetotail current sheets, Geophys. Res. Lett., 29(12), doi:10.1029/ 2001GL013789, 2002.

M. André, Swedish Institute of Space Physics, Uppsala, Sweden.

A. Balogh, Imperial College, London, UK.

W. Baumjohann, R. Nakamura, M. Volwerk, and T. L. Zhang, Institut für Weltraumforschung der ÖAW, Graz, Austria.

B. Klecker, Max-Planck-Institut für extraterrestrische Physik, Garching, Germany.

H. Rème and J. A. Sauvaud, CESR/CNRS, Toulouse, France.

A. Runov, Institut für Weltraumforschung der ÖAW, Schmiedlstr. 6, A8042, Graz, Austria. (Andrei.Runov@oeaw.ac.at)

V. Sergeev, Institute of Physics, St. Petersburg University, St. Petersburg, Russia. 